\title{
Spot Size Dependence of Laser Accelerated Protons in Thin Multi-Ion Foils
}

\section{Tung-Chang Liu', Xi Shao ${ }^{1}$, Chuan-Sheng Liu ${ }^{1}$, Bengt Eliasson ${ }^{1,2}$, Jyhpyng Wang ${ }^{3,4}$ and Shih-Hung Chen ${ }^{4}$}

${ }^{1}$ Department of Physics, University of Maryland, College Park, Maryland 20742, USA

${ }^{2}$ SUPA, Department of Physics, Strathclyde University, Glasgow G4 0NG, United Kingdom

${ }^{3}$ Institute of Atomic and Molecular Sciences, Academia Sinica, Taipei 10617, Taiwan

${ }^{4}$ Department of Physics, National Central University, Taoyuan 32001, Taiwan

E-mail: tcliu@umd.edu

\begin{abstract}
We present a numerical study of the effect of the laser spot size of a circularly polarized laser beam on the energy of quasi-monoenergetic protons in laser proton acceleration using a thin carbon-hydrogen foil. The used proton acceleration scheme is a combination of laser radiation pressure and shielded Coulomb repulsion due to the carbon ions. We observe that the spot size plays a crucial role in determining the net charge of the electron-shielded carbon ion foil and consequently the efficiency of proton acceleration. Using a laser pulse with fixed input energy and pulse length impinging on a carbon-hydrogen foil, a laser beam with smaller spot sizes can generate higher energy but fewer quasi-monoenergetic protons. We studied the scaling of the proton energy with respect to the laser spot size and obtained an optimal spot size for maximum proton energy flux. Using the optimal spot size, we can generate an $80 \mathrm{MeV}$ quasi-monoenergetic proton beam containing more than $10^{8}$ protons using a laser beam with power $250 \mathrm{TW}$ and energy $10 \mathrm{~J}$ and a target of thickness 0.15 wavelength and 49 critical density made of $90 \%$ carbon and $10 \%$ hydrogen.
\end{abstract}

\section{Introduction}

Since the initial prediction ${ }^{1}$ and experimental verifications ${ }^{2-4}$ of the laser wake-field acceleration of electrons, there has been an active research on advancing laser particle acceleration schemes for a wide variety of applications. Laser-plasma based acceleration schemes ${ }^{5}$ allow for a revolutionary reduction of the size of accelerators as the acceleration gradient provided by terawatt to petawatt lasers can reach as high as hundreds of $\mathrm{GeV}$ per $\mathrm{cm}$. In particular, the laser acceleration of quasi-monoenergetic protons has recently drawn tremendous interests due to its potential applications in cancer treatment. ${ }^{6,7}$ In the realm of laser acceleration of protons from a target foil, there are mainly two schemes being widely studied, target normal sheath acceleration (TNSA) and radiation pressure acceleration (RPA). In TNSA, the laser irradiates foils with thicknesses tens of 
laser wavelengths, and the protons are accelerated to tens of $\mathrm{MeV}$ by the electric field created by the hot electrons heated by the laser. ${ }^{8-17}$ However, in most cases, the resulting ion energy spectra are broad and only few protons reach the maximum energy, which makes TNSA less suitable for applications requiring monoenergetic protons.

In order to acquire quasi-monoenergetic protons, the scheme of laser RPA has been actively studied in theory and simulations ${ }^{18-26}$ and experiments. ${ }^{27,28}$ In RPA, a high intensity circularly polarized laser beam irradiates an ultra-thin foil and accelerates nearly the whole foil by the radiation pressure. The electrons are trapped by a combination of the laser ponderomotive force and the electric force due to the ions, and the protons in the accelerating frame are subject to both the electric force of the electron layer accelerating them forward and the inertial force pulling them back. The balance of these opposing forces forms a trap for the proton and electron layers, resulting in a self-organized double layer. ${ }^{24}$ Therefore, RPA is a more efficient acceleration process for producing high energy monoenergetic protons, suitable for many applications requiring that the accelerated protons have good beam quality and a narrow energy spectrum. However, previous works demonstrated ${ }^{23,25,29-31}$ that the Rayleigh-Taylor instability (RTI) limits the acceleration achieved by RPA and rapidly broadens the proton beam's energy spectrum.

For the RPA of thin-foil targets of only protons, our energy scaling study with twodimensional (2D) particle-in-cell (PIC) simulations ${ }^{25}$ indicates that petawatt power laser is required to obtain $\sim 200 \mathrm{MeV}$ quasi-monoenergetic protons with the full-width-half-maximum energy spread less than $20 \%$ of the peak flux energy, which may reduce the attractiveness of the laser proton acceleration scheme for commercial practical applications as it is difficult to build and yields a highly radioactive environment. On the other hand, by using a thin composite foil made of carbon and hydrogen, our recent work ${ }^{32}$ found that there are two different stages of acceleration to further push the proton forward. The first one is the RPA stage, in which the heavier carbon ions are less accelerated and are left behind the lighter protons, forming a triple layer system. After the electron layer is disrupted by the RTI, the shielded Coulomb repulsion (SCR) stage takes place, in which the proton layer continues to be pushed forward by the electron-shielded carbon ion layer behind. The carbon layer can delay the disruption of the proton layer by the RTI and further accelerate the protons. Using a 70 terawatt laser beam to irradiate a carbon-proton target with $10 \%$ protons, a quasi-monoenergetic proton beam with $60 \mathrm{MeV}$ of energy was achieved, which is several times the energy obtained from a pure hydrogen foil.

In the SCR stage of the laser acceleration of protons from a multi-ion foil, there are two crucial factors deciding the acceleration efficiency, the carbon-proton ratio and the laser spot size. Clearly the total charge of the electrons in a neutralized foil is always greater than that of the carbon ions, and therefore, after the electron layer become transparent to the laser light and cease to be pushed by the radiation pressure, a majority of the electrons will return to the carbon layer and slow down the acceleration of the protons. Therefore, in order to successfully accelerate the protons, we should both reduce the charge difference between carbon ions and electrons, and keep the electrons from returning to the carbon layer. Our previous work ${ }^{32}$ studied the carbon-proton ratio and concluded that a higher carbon concentration leads to an increased proton energy. In this paper, we focus on the effect of the spot size. We present that a small spot size can help to repel the electrons in the transverse direction and keep them away from the central axis of the laser beam, generating an environment with abundant positive charges due to the carbon ions. Using a laser pulse with power $250 \mathrm{TW}$ and energy $10 \mathrm{~J}$ and a target of thickness 0.15 wavelength with a density 49 times the critical density made of $90 \%$ carbon and $10 \%$ hydrogen, we can obtain a beam of 
quasi-monoenergetic protons with energies exceeding $100 \mathrm{MeV}$, which is promising for medical applications.

\section{Simulation Setup}

In order to investigate the acceleration of protons in a multi-ion foil for different laser spot sizes, we employ 2D PIC simulations. The simulation domain is $-50 \leq x / \lambda_{\mathrm{L}} \leq 100$ and $-25 \leq y / \lambda_{\mathrm{L}} \leq 25$, and the grid size is $\lambda_{\mathrm{L}} / 100$ in $x$ dimension and $\lambda_{\mathrm{L}} / 50$ in $y$ dimension, where $\lambda_{\mathrm{L}}=800 \mathrm{~nm}$ is the laser wavelength. The boundary conditions are absorbing at all boundaries for particles and fields, and the laser wave is injected at the negative $x$-boundary. The foil, consisting of $90 \%$ carbon and $10 \%$ hydrogen, is initially located at $0 \leq x \leq l_{0}$ with initial thickness $l_{0}=0.15 \lambda_{\mathrm{L}}$ and electron density $n_{\mathrm{e} 0}=49 n_{\mathrm{cr}}$ and is resolved by 100 macro-particles of each species per cell. Here $n_{\text {cr }}=\varepsilon_{0} m_{\mathrm{e}} \omega_{\mathrm{L}}^{2} / e^{2}$ is the critical density, where $m_{\mathrm{e}}$ is the mass of an electron, $e$ is the elementary charge, $\varepsilon_{0}$ is the vacuum permittivity and $\omega_{\mathrm{L}}$ is the laser angular frequency. The amplitude of the incident laser has a Gaussian profile in the transverse direction with spot size, defined as the diameter $d=2 w_{0}$ at $e^{-2}$ of the peak intensity at $x=0$, and a halfsine wave in time profile with a full duration of $30 T_{\mathrm{L}}$, where $w_{0}$ and $T_{\mathrm{L}}=\lambda_{\mathrm{L}} / c_{0}$ are respectively the waist size and the wave period. We define $t=0$ as the time when the laser beam starts to interact with the target.

\section{Simulation Result and Analysis}

We first compare the simulation results using two different spot sizes being $w_{0}=5.0 \lambda_{\mathrm{L}}$ and $w_{0}=7.5 \lambda_{\mathrm{L}}$, where the input laser peak amplitudes for the two cases are $a_{0}=15$ and $a_{0}=10$, respectively, such that the total power of the input laser is kept identical for the two cases. Figure 1 shows the comparison of the density distribution of each species in these two cases at $t=30 T_{\mathrm{L}}$, after that the laser pulses have interacted with the foil. At this time, the foil has moved further for the case with smaller spot size and larger peak amplitude, due to the larger ponderomotive force. On the other hand, with larger spot size, the foil moves more uniformly and maintains the layer structure for a longer time. We also observe that the typical size of the density perturbation in the transverse direction due to the RTI is smaller in the case with larger spot size, which is consistent with our previous work ${ }^{25}$ indicating that a smaller laser amplitude results in larger saturation wavenumber $k_{\mathrm{s}}$. Figure 2 shows the density distributions of each species together with the electrostatic field at the center axis at the $t=30 T_{\mathrm{L}}$. Since the electrons in the case of larger laser amplitude and smaller spot size are further spread out in both longitudinal and transverse directions, the net positive charge of the carbon-electron layer is larger, providing a greater electrostatic force pushing the proton layer forward compared to the case with smaller amplitude and larger spot size. The reason that the electrons are further spread out in longitudinal direction is because a larger input laser amplitude can result in higher electron temperature, ${ }^{32}$ whereas a further electron spread in transverse direction is due to a larger gradient in the laser ponderomotive force. Since these factors both positively contribute to the electrostatic field near the central axis, a more focused laser pulse can significantly increase the acceleration of the proton layer. 

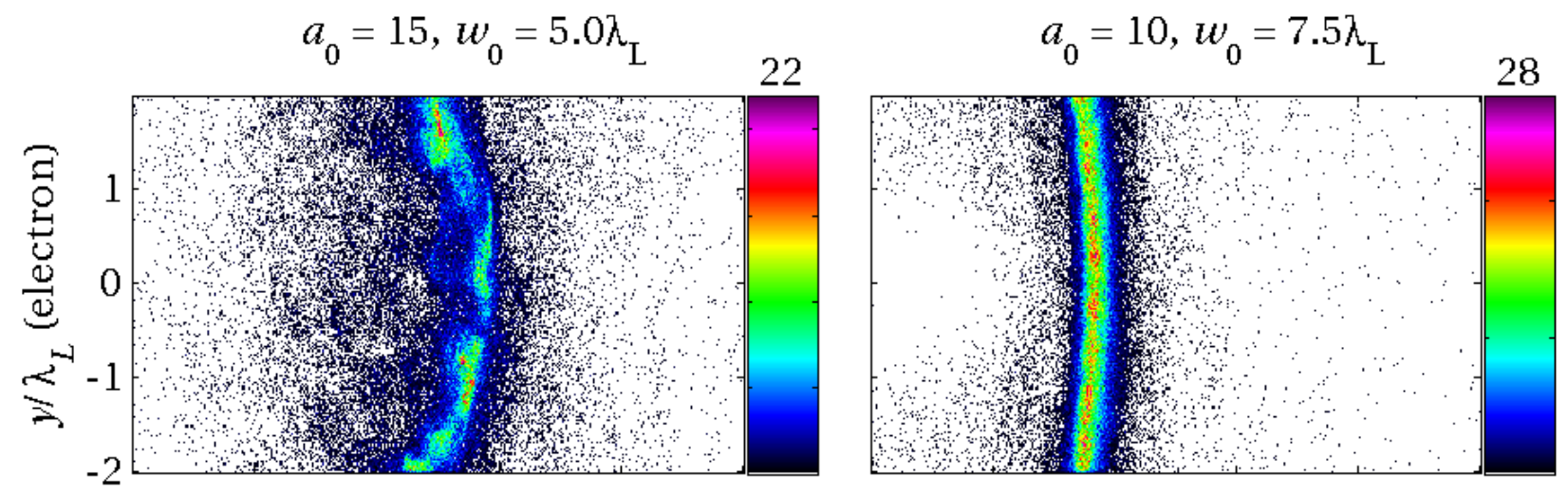

35
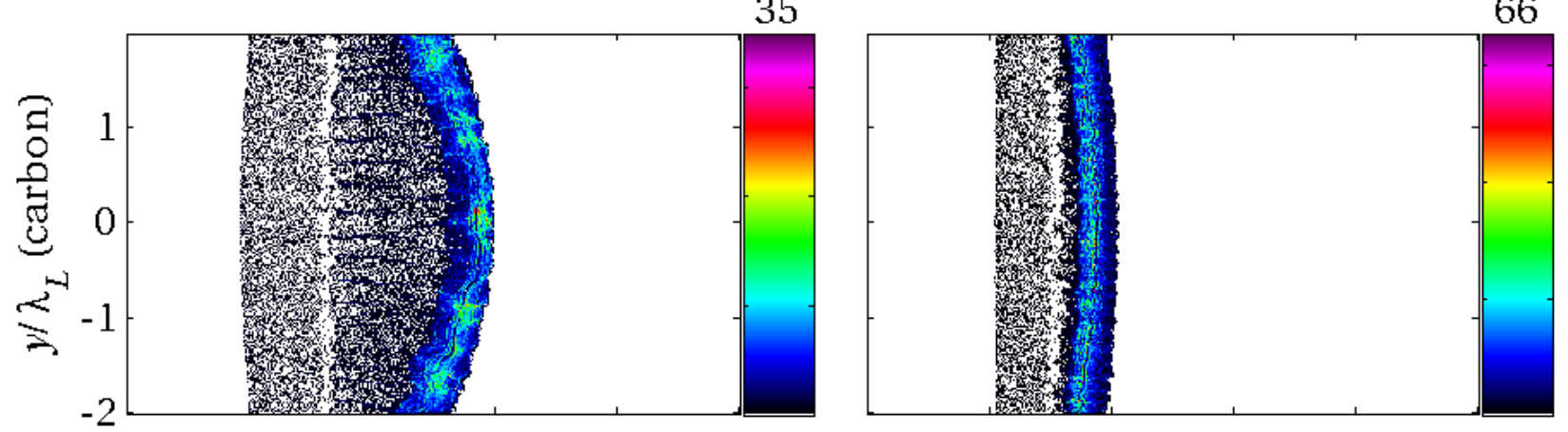

5.9

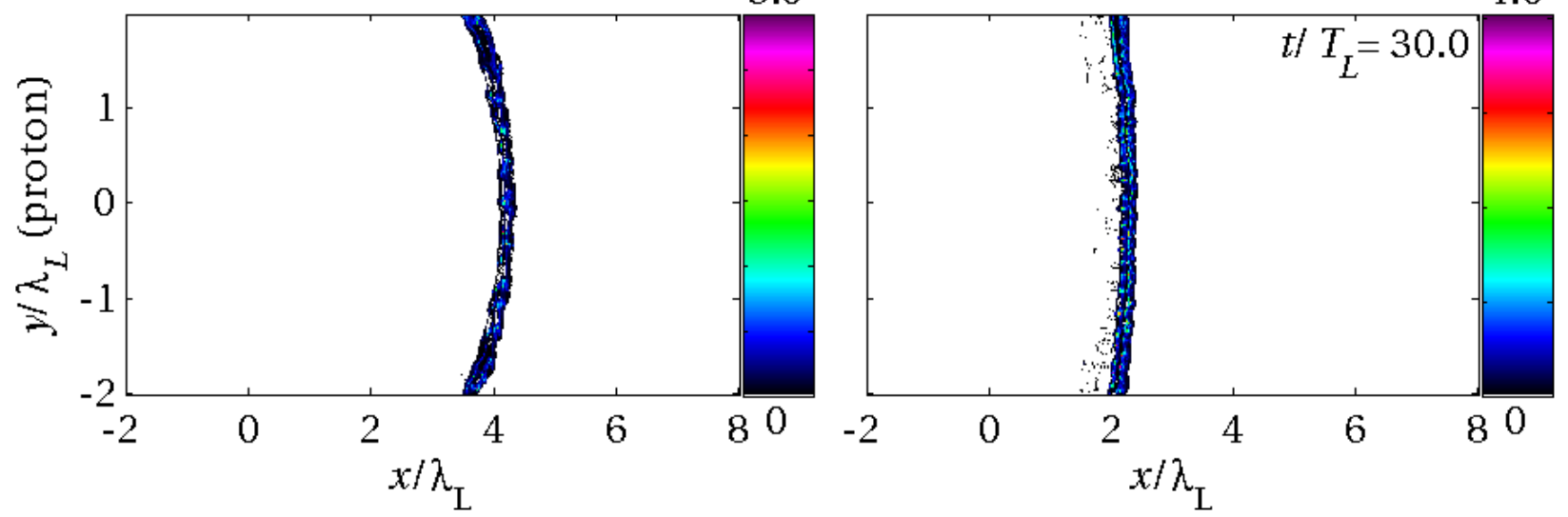

Figure 1: The density distribution of electrons, carbon ions and protons (the $1^{\text {st }}, 2^{\text {nd }}$ and $3^{\text {rd }}$ rows, respectively) in the cases with input laser parameters being respectively $a_{0}=15$, $w_{0}=5.0 \lambda_{\mathrm{L}}$ (the $1^{\text {st }}$ column) and $a_{0}=10, w_{0}=7.5 \lambda_{\mathrm{L}}$ (the $2^{\text {nd }}$ column) at $t=30 T_{\mathrm{L}}$. 

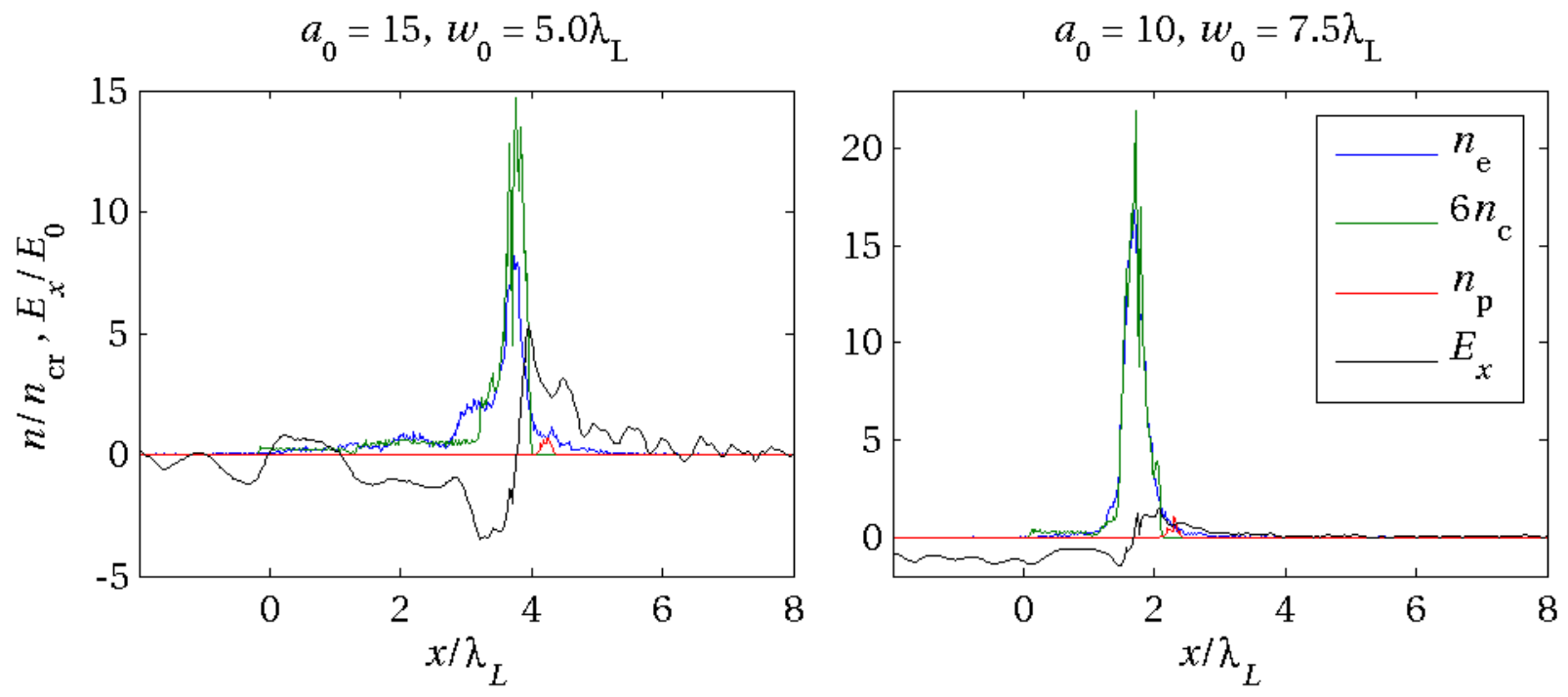

Figure 2: The density distributions at $t=30 T_{\mathrm{L}}$ of electrons $\left(n_{\mathrm{e}}\right)$, carbon ions $\left(n_{\mathrm{c}}\right)$ and protons $\left(n_{\mathrm{p}}\right)$ and the electrostatic field $\left(E_{x}\right)$ at the center axis with the same parameters as in Figure 1.

Although using a more focused laser beam can significantly increase the acceleration of the proton layer and consequently the energy of the quasi-monoenergetic peak, the disadvantage with a too large transverse gradient in laser intensity is the resulting transverse electric field drives away the protons in the transverse direction as well. Therefore, the number of protons accelerated along the central axis is decreased with reduced spot size. Since the shape of carbon-electron layer is also affected by the laser spot size, this effect becomes more and more destructive with time even after the laser pulse has passed. Figure 3 shows the density distribution of the charged particles at $t=100 T_{\mathrm{L}}$ when the laser pulses has long passed the foil. Two observations can be made at the later stage of the acceleration process. On one hand, during the time that the laser pulse no longer interacts with the foil, the electrons will gradually return to the carbon layer due to the attractive force, resulting in a reduction in proton acceleration. We observed that the proton acceleration for both cases is significantly smaller at $t=100 T_{\mathrm{L}}$ than during the first 30 wave periods. On the other hand, the evolution of the density distributions are very different. The charged particles in the case with smaller laser spot size are blown out more thoroughly so that the surface number density of the proton layer is less than half of that with larger spot size. Therefore, if we define the energy conversion efficiency as the total energy of the proton layer divided by the input laser energy, a more focused laser beam may not always lead to a higher efficiency. 

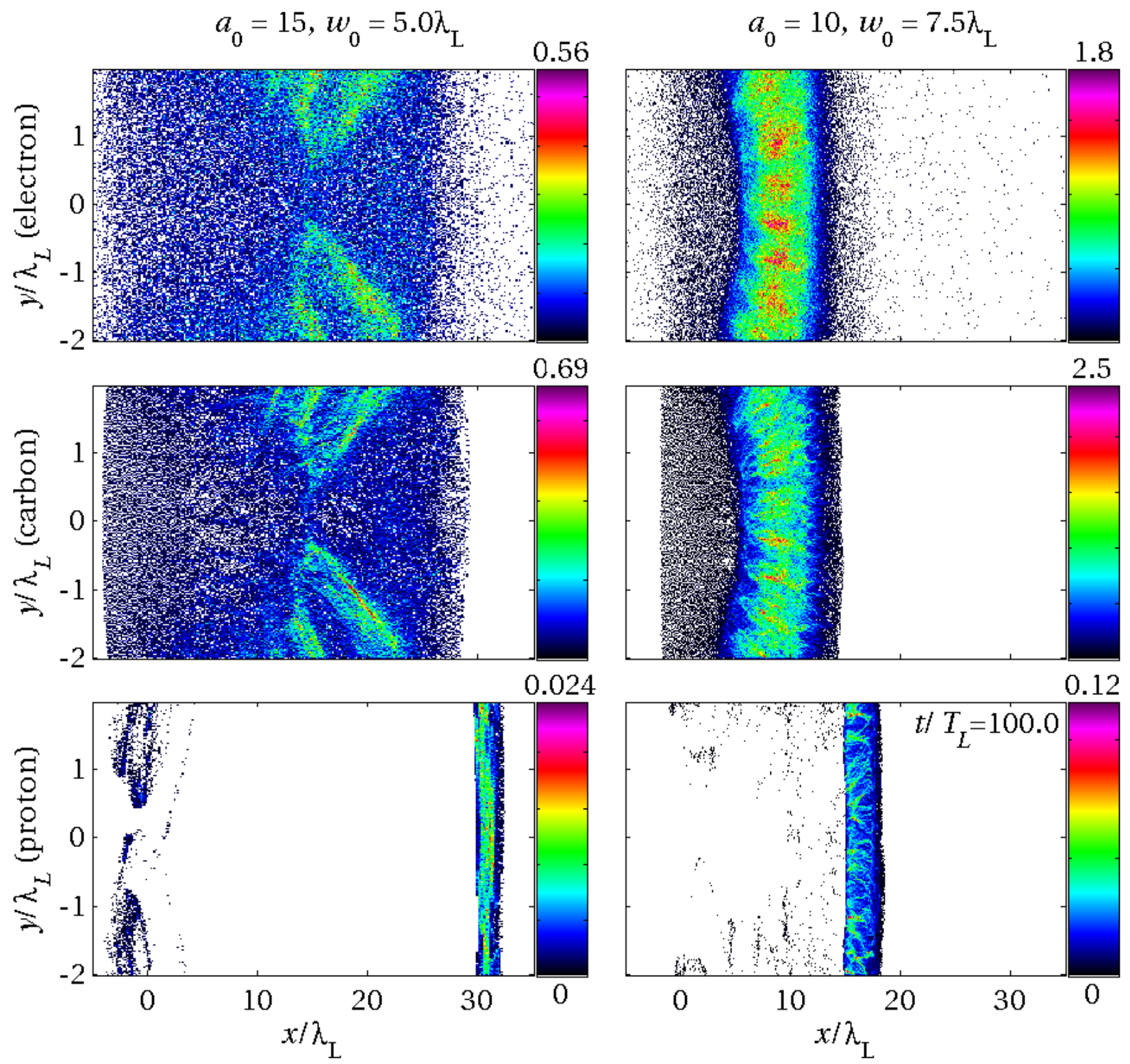

Figure 3: The distribution of the charged particles at $t=100 T_{\mathrm{L}}$ for the same parameters as in Figure 1. 


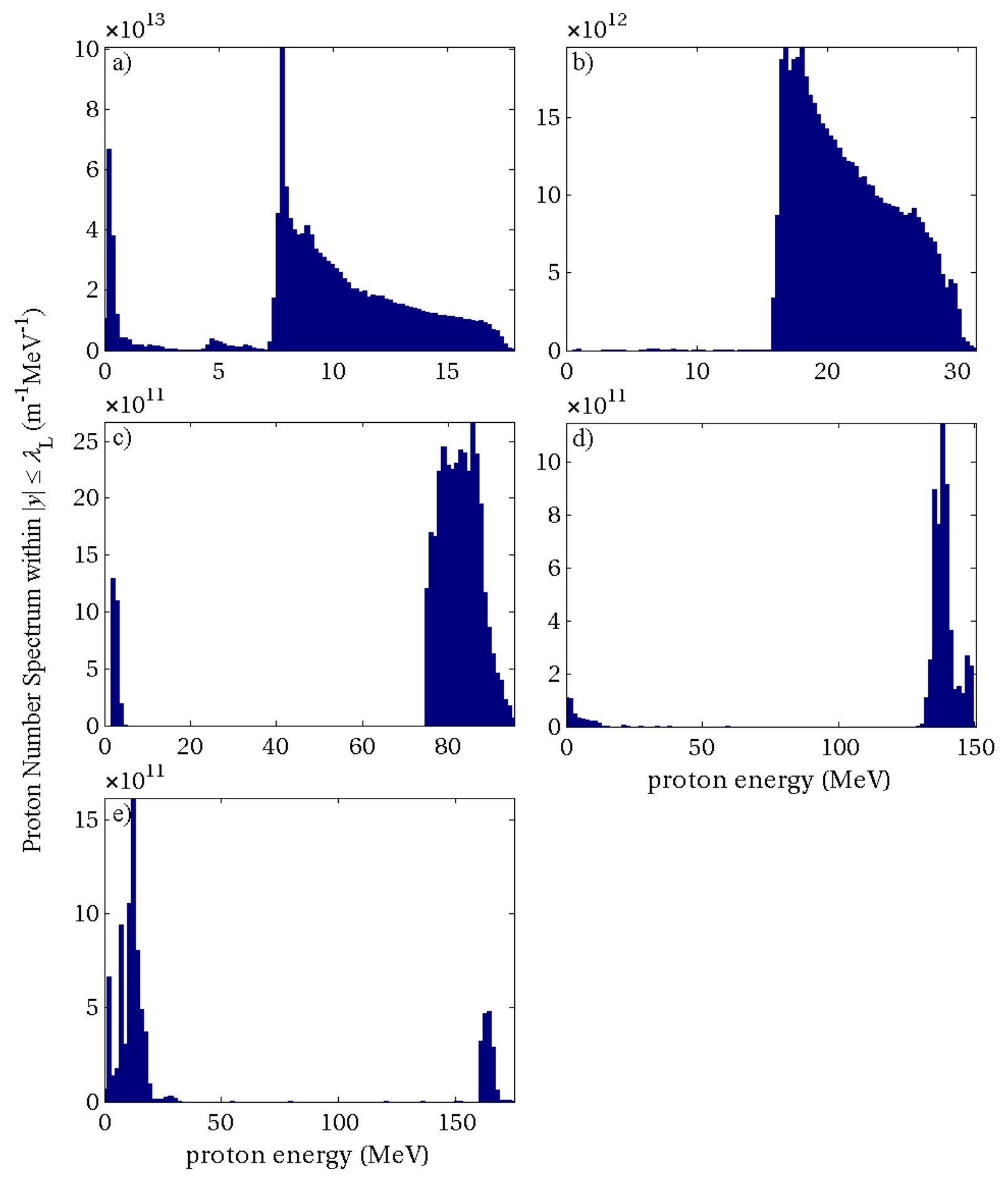

Figure 4: The proton energy spectra with different laser spot sizes at $t=100 T_{\mathrm{L}}$. The input parameters are (a) $a_{0}=7.6, w_{0}=10 \lambda_{\mathrm{L}}$, (b) $a_{0}=10, w_{0}=7.5 \lambda_{\mathrm{L}}$, (c) $a_{0}=15, w_{0}=5.0 \lambda_{\mathrm{L}}$, (d) $a_{0}=30, w_{0}=2.5 \lambda_{\mathrm{L}}$ and $(\mathrm{e}) a_{0}=76, w_{0}=1.0 \lambda_{\mathrm{L}}$. 


\section{Scaling}

Two questions arise from the observations described above. On one hand, since the proton acceleration with large laser spot size is evidently smaller, it is of interest whether there is a criterion for an optimal laser spot size for quasi-monoenergetic acceleration of the protons. Since the Coulomb repulsion is based on the positive net charge of the carbon-electron layer due to that a portion of the electrons are ejected from the center part of the carbon layer, using a laser beam with too large spot size may not force the electrons to escape transversely and result in a more neutralized carbon-electron layer. Noticing that the initial charge number of the electrons is actually higher than the total charge of the carbon ions, the carbon-electron layer can easily be totally neutralized if the laser beam fails to push away a large enough amount of electrons from the carbon layer. In this case, the proton layer is no longer accelerated by the SCR process. Therefore, it is crucial to find the criterion of the laser spot size so that the proton layer can be successfully accelerated by SCR. Figure 4 shows a comparison of proton energy spectra for different spot sizes ranging from $w_{0}=10 \lambda_{\mathrm{L}}$ (a) to $w_{0}=1 \lambda_{\mathrm{L}}(\mathrm{e})$. With a large laser spot size, the protons are less accelerated, and the energy spectrum is more broadened as well. We can observe in Figure 4(c)-4(e) that a spot size of $w_{0} \leq 5 \lambda_{\mathrm{L}}$ is required to have the full-width-half-maximum (FWHM) spread in proton energy within $15 \%$. Moreover, although with fewer protons, $160 \mathrm{MeV}$ of quasi-monoenergetic protons are obtained in this acceleration scheme. On the other hand, it is also important to explore the maximum of the energy conversion efficiency among different spot sizes. Figure 5(a)-6(c) show the evolution of average proton energies in the quasi-monoenergetic peaks, total proton numbers and total proton energies near the center axis among different input laser spot sizes. In Figure 5(a), in additional to the fact we have discussed above that the energy is negatively relative to the laser spot size, we can also see that the acceleration of protons for $t>30 T_{\mathrm{L}}$ gradually reaches its saturation value, which is a similar effect regardless of the input spot sizes, showing that the acceleration by the SCR decreases with increasing distance between the proton and carbon-electron layers. Moreover, the proton energy spectra at $t=100 T_{\mathrm{L}}$ shown in Figure 4 also indicate that the protons accelerated with a small enough laser spot size can maintain their quasi-monoenergetic properties for a long time even after the acceleration is saturated [see Figure 5(a)], suggesting that SCR is a stable acceleration scheme. In contrast, Figure 5(b) shows that the number of protons we can obtain decreases drastically when reducing the laser spot size. For example, the number of protons in the quasi-monoenergetic peak in the case with spot size $w_{0}=1 \lambda_{\mathrm{L}}$ is less than $10 \%$ of the number of protons in the $w_{0}=10 \lambda_{\mathrm{L}}$ case. Considering these two factors altogether in order to explore the condition for optimal energy conversion efficiency, we can then observe from Figure 5(c) that $w_{0}=5 \lambda_{\mathrm{L}}$ is an optimal value of conversion efficiency over these spot size values. The conversion efficiency is here defined by the total energy of the proton beam within the range of $|y|<\lambda_{\mathrm{L}}$ and $|z|<\lambda_{\mathrm{L}}$ divided by the input laser energy. 


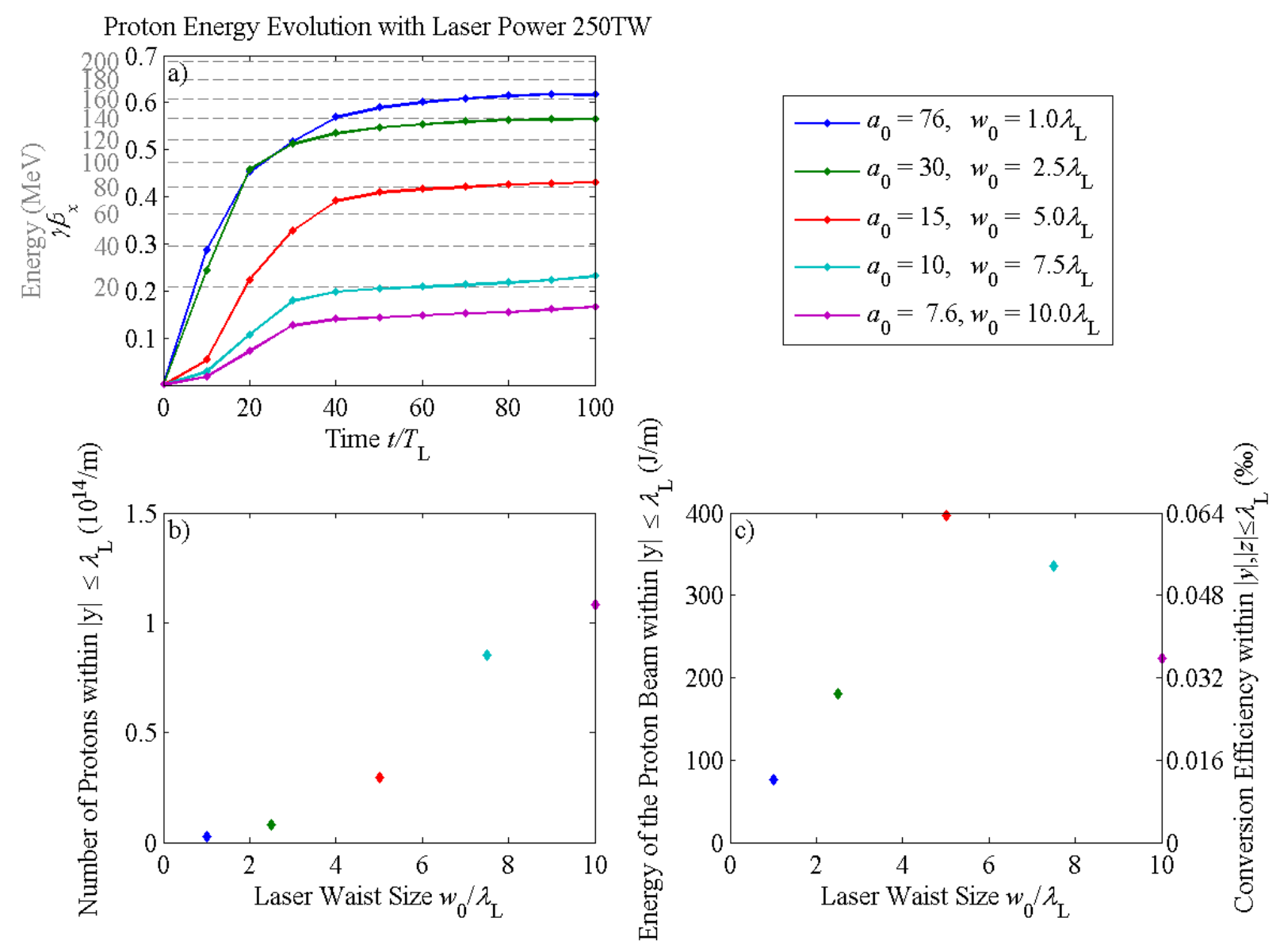

Figure 5: The comparison of (a) the evolution of proton momentum, (b) the proton number and (c) the proton energy flux and conversion efficiency among different input laser spot sizes.

\section{Discussions}

In this section, we provide a simple model interpreting the scaling of the energy, number and total energy flux of protons with different spot sizes based on RPA theory since it is the dominant acceleration mechanism. The energy gain due to SCR, as shown in $t>30 T_{\mathrm{L}}$ region of Figure 5(a), is relatively insignificant. For the RPA theory to be valid, we need to first check if the foil can maintain its overdense property so that the laser beam can be fully reflected during the pulse time. Figure 6 shows the electric fields in the longitudinal and one of the perpendicular directions, along with the density distributions from simulations of three different spot sizes at $t=30 T_{\mathrm{L}}$, near the end of the laser pulse. We can clearly observe that with larger spot size and smaller laser intensity [Figure 6(a)], the foil is still overdense and the laser pulse is nearly totally reflected, whereas with smaller spot size and larger laser intensity [Figure 6(c)], the foil becomes underdense. Therefore, with large laser amplitude $a_{0}$ such as that in Figure 6(c), the rest of the laser pulse will then pass through after the foil becomes transparent, and very little energy can be transferred to the particles. This explains that the total energy of the proton beam is reduced when the laser beam is too focused to keep the foil as overdense during the irradiation of the laser pulse 
on the foil. Therefore, we then focus on explaining the conditions to obtain maximal proton energy flux among cases without induced-transparency at $t=30 T_{\mathrm{L}}$.
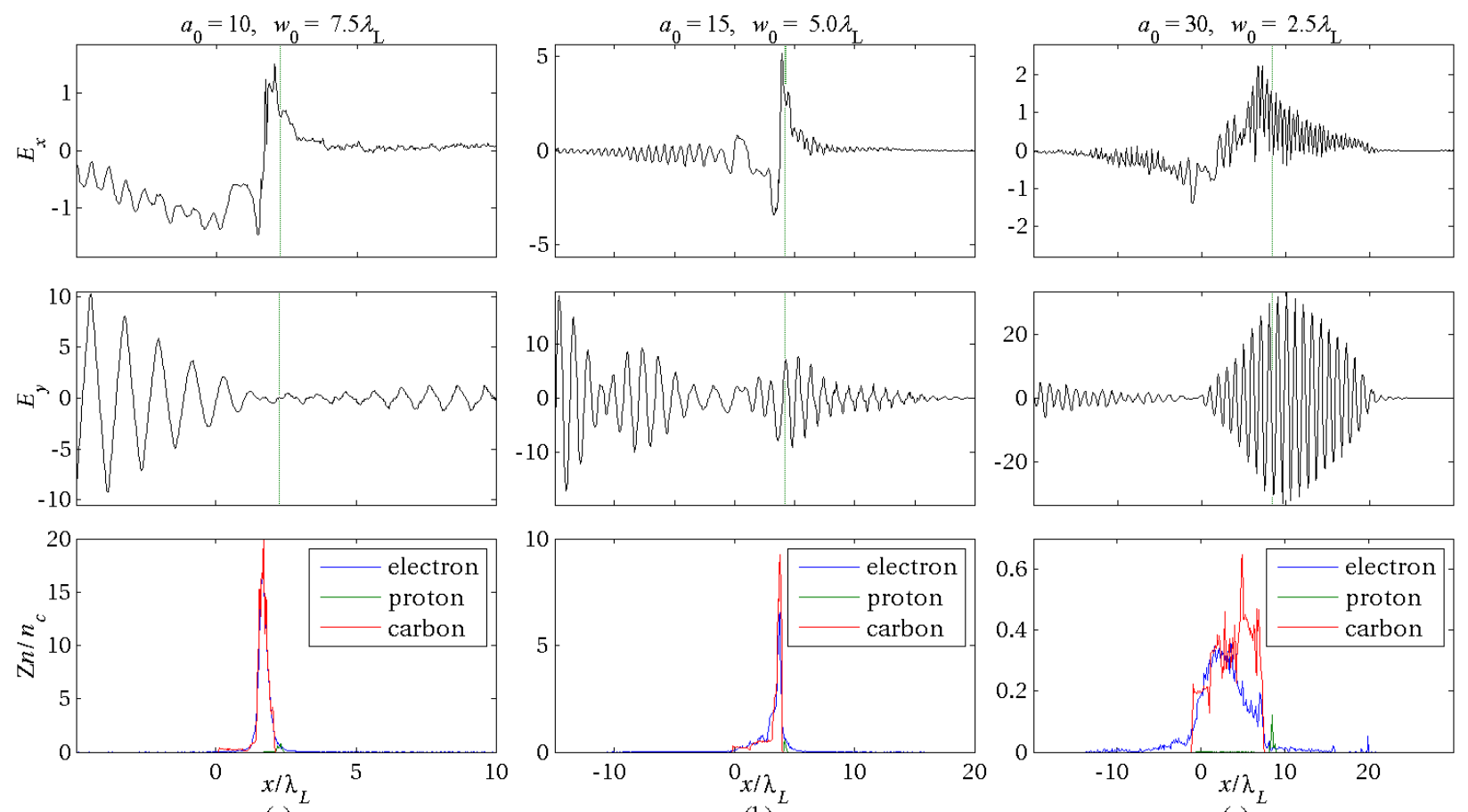

(a)

(b)

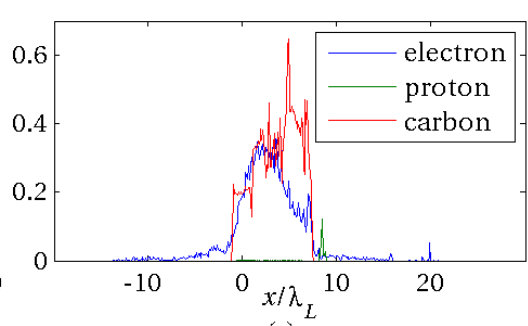

(c)

Figure 6: The comparison of $E_{x}$ (the first row), $E_{y}$ (the second row) and charge density distribution (the third row) at the center axes at $t=30 T_{\mathrm{L}}$ with $w_{0}=7.5 \lambda_{\mathrm{L}}, 5.0 \lambda_{\mathrm{L}}$ and $2.5 \lambda_{\mathrm{L}}$ from the first to the third columns, respectively.

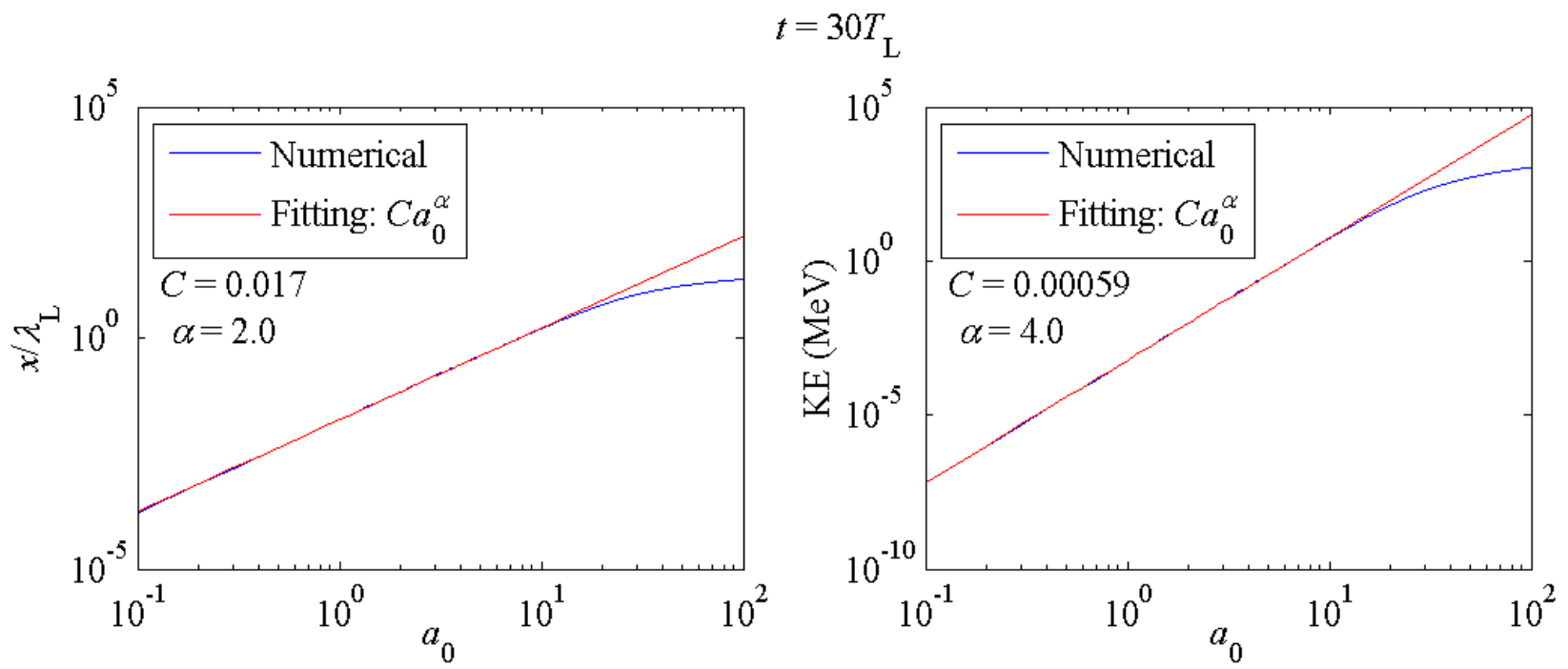

Figure 7: The theoretical scaling of foil position $x$ (left column) and proton energy (right column) to laser input amplitude $a_{0}$ at $t=30 T_{\mathrm{L}}$, along with the fitting in log scale. 
Next, we estimate how proton energy and number depend on the input spot size by calculating the transverse expansion of the foil around the center axis. The proton energy can be obtained by numerically solving the equations of motion of RPA, ${ }^{22}$ which are listed as

$$
\left\{\begin{aligned}
\frac{\mathrm{d} x_{\mathrm{i}}}{\mathrm{d} t} & =\beta_{\mathrm{i}} \\
\frac{\mathrm{d} \gamma_{\mathrm{i}} \beta_{\mathrm{i}}}{\mathrm{d}\left(t / T_{\mathrm{L}}\right)} & =2 \frac{m_{\mathrm{e}}}{m_{\mathrm{i}}} \frac{n_{\mathrm{cr}}}{n_{0}} \frac{\lambda_{\mathrm{L}}}{l_{0}} a\left(x_{\mathrm{i}}, t\right)^{2} \frac{1-\beta_{\mathrm{i}}}{1+\beta_{\mathrm{i}}}
\end{aligned}\right.
$$

where

$$
a\left(x_{\mathrm{i}}, t\right)=a_{0} \sin \left[-\frac{\pi}{\tau_{\mathrm{L}}}\left(\frac{x_{\mathrm{i}}}{\lambda_{\mathrm{L}}}-\frac{t}{T_{\mathrm{L}}}\right)\right],-\tau_{\mathrm{L}}<\frac{x_{\mathrm{i}}}{\lambda_{\mathrm{L}}}-\frac{t}{T_{\mathrm{L}}}<0
$$

is the instantaneous normalized amplitude of the laser at the foil with $\tau_{\mathrm{L}}=t_{\mathrm{L}} / T_{\mathrm{L}}=30$ being the normalized laser pulse length. Figure 7 shows the dependence of the displacement in the $x$ direction of the foil at $t=30 T_{\mathrm{L}}$ on $a_{0}$ by RPA, along with a fitting curve $C a_{0}^{\alpha}$ computed for small $a_{0}$ cases demonstrating the quadratic and quartic dependence in longitudinal displacement and kinetic energy of the proton layer, respectively. The remaining proton number is calculated based on foil stretching, where the foil is simplified as a square shape that the center part $\left(-w_{0}<y<w_{0}\right)$ of the foil is pushed forward by a distance of $x / \lambda_{\mathrm{L}}=C a_{0}^{\alpha}$, as shown in Figure 7. Considering the prescribed condition that the input laser power is kept as a constant, $w_{0} \propto 1 / a_{0}$, the expansion ratio of the foil is then

$$
\frac{L}{L_{0}}=1+\frac{x}{w_{0}}=1+C_{1} a_{0}^{3}
$$

where $C_{1} \approx 1 / 4500$ is the fitting constant, $L_{0}=2 w_{0}$ is the initial length of the foil within laser spot size and $L=2 x+2 w_{0}$ is the total length of the foil after stretched by the laser beam. Assuming the conservation of total particle number, $N L=N_{0} L_{0}$, we can calculate the particle number ratio at $t=30 T_{\mathrm{L}}$ as

$$
\frac{N}{N_{0}}=\frac{w_{0}}{x+w_{0}} \approx \frac{1}{C_{1} a_{0}^{3}+1} .
$$

This ratio is calculated and visualized in Figure 8, which qualitatively illustrates the concept. Since particles continue to escape due to SCR, the trend shown in Figure 8 is an overestimate. If we further assume that the center part of the proton layer continues to expand due to self-repulsion with a constant transverse velocity, i.e. $v_{y}(x, y, t)=v_{y}(y) \propto y$, resulting in exponential decaying in the number of particle per length, then we can extrapolate Eq. (4) to $t=100 T_{\mathrm{L}}$, as also shown in Figure 8 with great agreement with small $a_{0}$ cases. Here $t=100 T_{\mathrm{L}}$ is chosen since the proton beam at this time is stable with nearly zero acceleration (Figure 5) and insignificant energy spectrum broadening. For larger $a_{0}$, the actual laser acceleration time is decreased due to foil transparency and thus the number of proton could be slightly greater. 
Finally, we similarly calculate the energy of the foil due to RPA to find the scaling, as also shown in Figure 7. In addition, the proton energy could be obtained from the velocity $v_{\mathrm{p}}$ by $v=\int q E / m \mathrm{~d} t$, where $E$ is the electrostatic field induced by RPA. Since the proton concentration is very little, the center-of-mass velocity of the foil is approximately the velocity of the carbon ion layer, and thus the proton layer is assumed to move two times faster [dashed line in Figure 9(a)] than the foil [solid line in Figure 9(a)] due to its charge-to-mass ratio. The result presented in Figure 9(a) shows a good agreement again for small $a_{0}$ cases. Computing the overall proton energy scaling by multiplying these two factors together, we can find a maximum at $a_{0} \approx 15$ as shown in Figure 9(b). Although this calculation is not valid quantitatively for large $a_{0}$ cases, since reduced RPA duration is less efficient in the acceleration process, we could conclude that the condition of maximum efficiency can be achieved around $a_{0} \approx 15$ or equivalently $w_{0} \approx 5.0 \lambda_{\mathrm{L}}$.

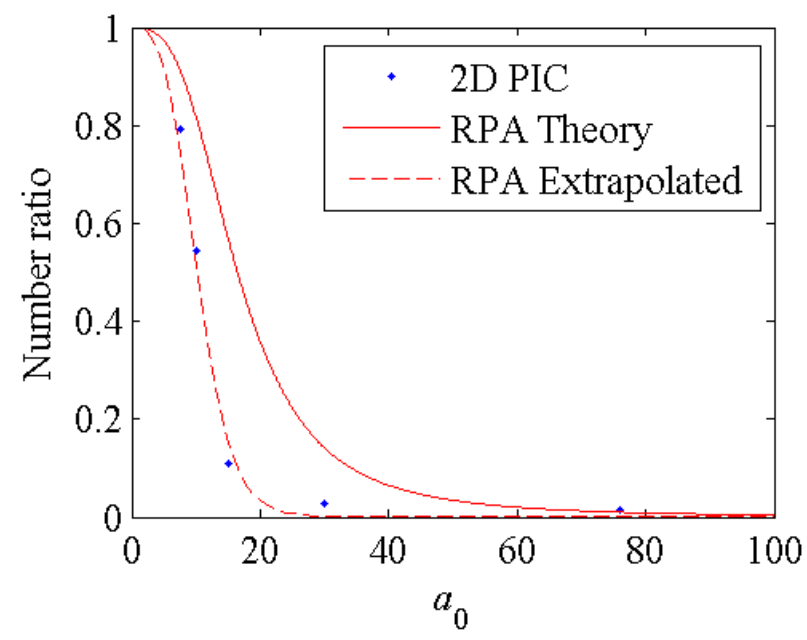

Figure 8: The scaling of simulated proton number ratio $N_{\mathrm{p}} / N_{\mathrm{p} 0}$ at $t=100 T_{\mathrm{L}}$ vs. laser input amplitude $a_{0}$, along with the theoretical approximation. The dashed line is the theoretical calculation extrapolated from $t=30 T_{\mathrm{L}}$ to $t=100 T_{\mathrm{L}}$
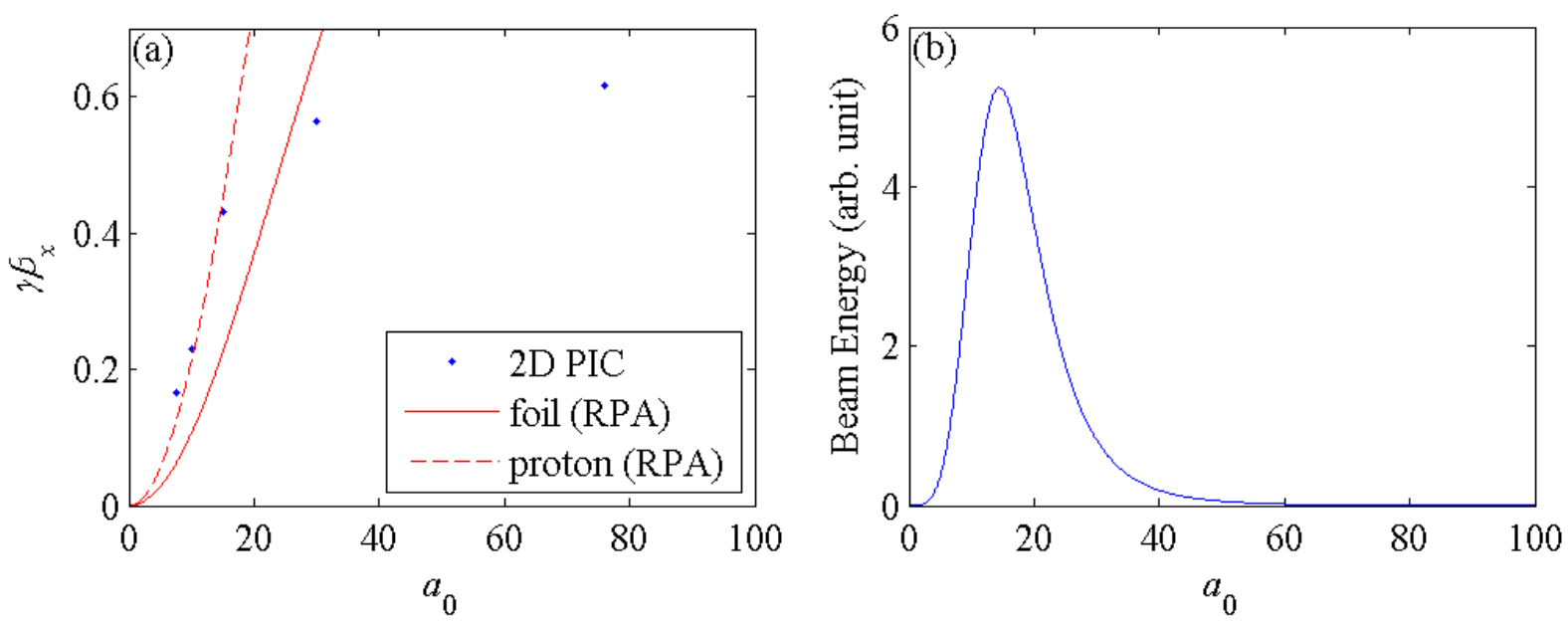
Figure 9: (a) The scaling of normalized proton velocity to laser input amplitude $a_{0}$, along with the theoretical approximation. The dashed line is calculated by assuming that the acceleration of the proton layer is two times the acceleration of the carbon foil. (b) The total proton beam energy obtained by multiplication of the particle number and the average proton energy.

Before concluding, here we discuss the issue of the critical density lower than in typical solids due to our limited computational resources. Since a higher initial particle density results in a lower $c / \omega_{\mathrm{pe}}$ value the problem should be resolved by a finer simulation grid, indicating a requirement of greater computational resource. In our current setting, the grid size is $\lambda_{\mathrm{L}} / 50$ and the initial $c / \omega_{\mathrm{pe}}$ value is $c / \omega_{\mathrm{pe} 0}=2 \pi \lambda_{\mathrm{L}} / 49=\lambda_{\mathrm{L}} / 7.8$. Assuming a maximum density compression ratio of 3 (which is roughly the case observed in the simulations), the density distribution of the foil is resolved by two grid points per $c / \omega_{\mathrm{pe}}$. In comparison, we also ran a case with doubled initial density $n_{\mathrm{e} 0}=98 m_{\mathrm{cr}}$ and halved foil thickness $l_{0}=0.075 \lambda_{\mathrm{L}}$, roughly the limiting case of resolving the density distribution by one grid per $c / \omega_{\text {pe }}$ with the same compression assumption. We observe that the proton momenta and the proton number do not change significantly due to the value of foil surface density being kept identical, as shown in Figure 10. We therefore believe that the conclusion will not change significantly if the initial density is increased 4 times to about $200 n_{\text {cr }}$, near the lower bound of solid density, while decreasing the initial thickness of the foil by a factor of 4 .
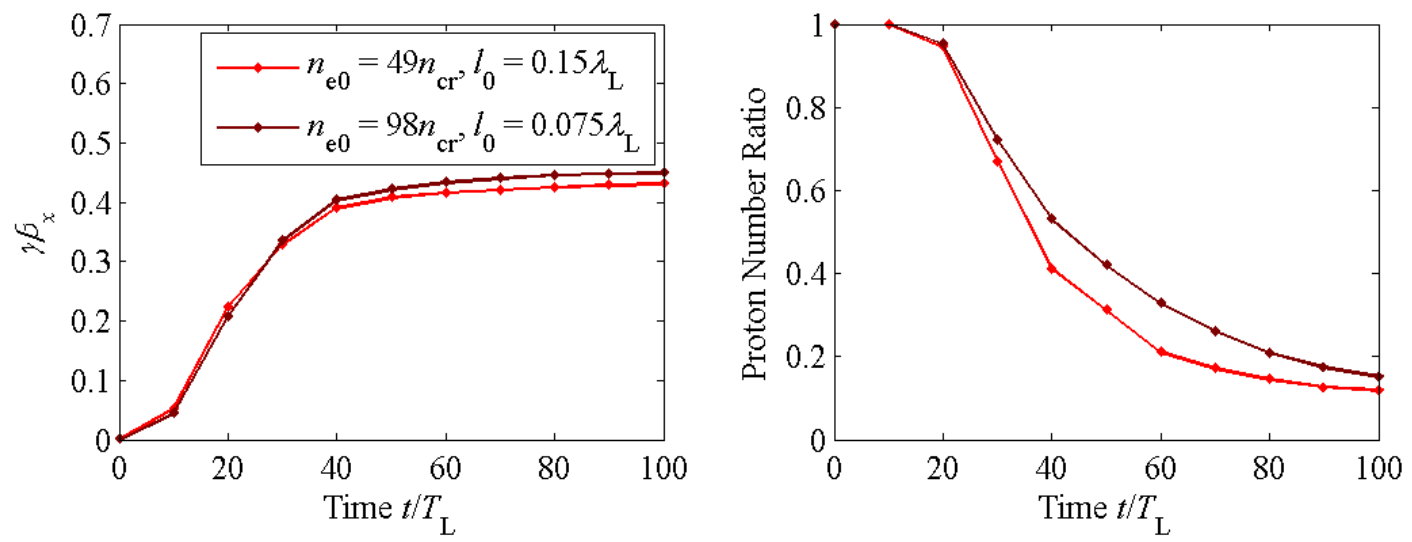

Figure 10: The comparison of the evolution of proton momenta (left column) and proton number ratio $N_{\mathrm{p}} / N_{\mathrm{p} 0}$ (right column) between the original case of $a_{0}=15, n_{\mathrm{e} 0}=49 n_{\mathrm{cr}}$, $l_{0}=0.15 \lambda_{\mathrm{L}}$ and the case with identical laser profile, doubled target density and halved target thickness.

\section{Conclusions}

We have numerically demonstrated the effects of different spot sizes on the two-stage acceleration schemes, RPA and SCR, using a finite circularly polarized laser pulse with a full duration of $30 T_{\mathrm{L}}$, peak power of $250 \mathrm{TW}$ and total energy of $10 \mathrm{~J}$ irradiated on a carbon-hydrogen 
thin foil with thickness $0.15 \lambda_{\mathrm{L}}$ and electron density $49 n_{\text {cr }}$ made of $90 \%$ carbon and $10 \%$ hydrogen. We compared cases with different laser spot sizes and observed that the optimal value of the spot size is about $w_{0}=5 \lambda_{\mathrm{L}}$ for maximal energy conversion efficiency. Using this scheme, $\sim 10^{7}$ quasimonoenergetic protons with $160 \mathrm{MeV}$ energy (the maximal proton energy case) or about $\sim 10^{8}$ quasi-monoenergetic protons with $80 \mathrm{MeV}$ energy (the maximal efficiency case) can be obtained with moderate laser input power and energy, which is promising for future applications.

\section{Acknowledgements}

This work was supported by US DoE grant DE-SC0008391. We acknowledge the National Center for High-Performance Computing for providing resources under the national project 'Taiwan Knowledge Innovation National Grid'. 


\section{References}

${ }^{1}$ T. Tajima and J.M. Dawson, Phys. Rev. Lett. 43, 267 (1979).

${ }^{2}$ S.P.D. Mangles, C.D. Murphy, Z. Najmudin, A.G.R. Thomas, J.L. Collier, A.E. Dangor, E.J. Divall, P.S. Foster, J.G. Gallacher, C.J. Hooker, D.A. Jaroszynski, A.J. Langley, W.B. Mori, P.A. Norreys, F.S. Tsung, R. Viskup, B.R. Walton, and K. Krushelnick, Nature 431, 535 (2004).

${ }^{3}$ C.G.R. Geddes, C. Toth, J. van Tilborg, E. Esarey, C.B. Schroeder, D. Bruhwiler, C. Nieter, J. Cary, and W.P. Leemans, Nature 431, 538 (2004).

${ }^{4}$ J. Faure, Y. Glinec, A. Pukhov, S. Kiselev, S. Gordienko, E. Lefebvre, J.-P. Rousseau, F. Burgy, and V. Malka, Nature 431, 541 (2004).

${ }^{5}$ R. Bingham, J.T. Mendonça, and P.K. Shukla, Plasma Phys. Control. Fusion 46, R1 (2004).

${ }^{6}$ B. Jones, BMJ 330, 979 (2005).

${ }^{7}$ K.W.D. Ledingham, W. Galster, and R. Sauerbrey, Br. J. Radiol. 80, 855 (2007).

8 S.C. Wilks, A.B. Langdon, T.E. Cowan, M. Roth, M. Singh, S. Hatchett, M.H. Key, D. Pennington, A. MacKinnon, and R.A. Snavely, Phys. Plasmas 8, 542 (2001).

${ }^{9}$ A. Pukhov, Phys. Rev. Lett. 86, 3562 (2001).

${ }^{10}$ H. Schwoerer, S. Pfotenhauer, O. Jäckel, K.-U. Amthor, B. Liesfeld, W. Ziegler, R. Sauerbrey, K.W.D. Ledingham, and T. Esirkepov, Nature 439, 445 (2006).

${ }^{11}$ B.M. Hegelich, B.J. Albright, J. Cobble, K. Flippo, S. Letzring, M. Paffett, H. Ruhl, J. Schreiber, R.K. Schulze, and J.C. Fernández, Nature 439, 441 (2006).

${ }^{12}$ S. Ter-Avetisyan, M. Schnürer, P.V. Nickles, M. Kalashnikov, E. Risse, T. Sokollik, W. Sandner, A. Andreev, and V. Tikhonchuk, Phys. Rev. Lett. 96, 145006 (2006).

13 J. Fuchs, C.A. Cecchetti, M. Borghesi, T. Grismayer, E. d' Humières, P. Antici, S. Atzeni, P. Mora, A. Pipahl, L. Romagnani, A. Schiavi, Y. Sentoku, T. Toncian, P. Audebert, and O. Willi, Phys. Rev. Lett. 99, 015002 (2007).

${ }^{14}$ P. Mora, AIP Conf. Proc. 920, 98 (2007).

15 L. Yin, B.J. Albright, B.M. Hegelich, K.J. Bowers, K.A. Flippo, T.J.T. Kwan, and J.C. Fernández, Phys. Plasmas 14, 056706 (2007).

${ }^{16}$ L. Robson, P.T. Simpson, R.J. Clarke, K.W.D. Ledingham, F. Lindau, O. Lundh, T. McCanny, P. Mora, D. Neely, C.-G. Wahlström, M. Zepf, and P. McKenna, Nat. Phys. 3, 58 (2007).

${ }^{17}$ L. Yin, B.J. Albright, K.J. Bowers, D. Jung, J.C. Fernández, and B.M. Hegelich, Phys. Rev. Lett. 107, 045003 (2011).

18 T. Esirkepov, M. Borghesi, S.V. Bulanov, G. Mourou, and T. Tajima, Phys. Rev. Lett. 92, 175003 (2004).

${ }^{19}$ X.Q. Yan, C. Lin, Z.M. Sheng, Z.Y. Guo, B.C. Liu, Y.R. Lu, J.X. Fang, and J.E. Chen, Phys. Rev. Lett. 100, 135003 (2008).

${ }^{20}$ C.S. Liu, V.K. Tripathi, and X. Shao, AIP Conf. Proc. 1061, 246 (2008).

${ }^{21}$ O. Klimo, J. Psikal, J. Limpouch, and V.T. Tikhonchuk, Phys. Rev. Spec. Top. - Accel. Beams 11, 031301 (2008).

${ }^{22}$ V.K. Tripathi, C.S. Liu, X. Shao, B. Eliasson, and R.Z. Sagdeev, Plasma Phys. Control. Fusion 51, 024014 (2009).

${ }^{23}$ A.P.L. Robinson, M. Zepf, S. Kar, R.G. Evans, and C. Bellei, New J. Phys. 10, 013021 (2008).

${ }^{24}$ B. Eliasson, C.S. Liu, X. Shao, R.Z. Sagdeev, and P.K. Shukla, New J. Phys. 11, 073006 (2009).

${ }^{25}$ T.-C. Liu, X. Shao, C.-S. Liu, J.-J. Su, B. Eliasson, V. Tripathi, G. Dudnikova, and R.Z. Sagdeev, Phys. Plasmas 18, 123105 (2011). 
${ }^{26}$ M.-Q. He, X. Shao, C.-S. Liu, T.-C. Liu, J.-J. Su, G. Dudnikova, R.Z. Sagdeev, and Z.-M. Sheng, Phys. Plasmas 19, 073116 (2012).

${ }^{27}$ A. Henig, S. Steinke, M. Schnürer, T. Sokollik, R. Hörlein, D. Kiefer, D. Jung, J. Schreiber, B.M. Hegelich, X.Q. Yan, J. Meyer-ter-Vehn, T. Tajima, P.V. Nickles, W. Sandner, and D. Habs, Phys. Rev. Lett. 103, 245003 (2009).

${ }^{28}$ D. Jung, L. Yin, B.J. Albright, D.C. Gautier, R. Hörlein, D. Kiefer, A. Henig, R. Johnson, S. Letzring, S. Palaniyappan, R. Shah, T. Shimada, X.Q. Yan, K.J. Bowers, T. Tajima, J.C. Fernández, D. Habs, and B.M. Hegelich, Phys. Rev. Lett. 107, 115002 (2011).

${ }^{29}$ F. Pegoraro and S.V. Bulanov, Phys. Rev. Lett. 99, 065002 (2007).

${ }^{30}$ C.S. Liu, X. Shao, B. Eliasson, T.C. Liu, G. Dudnikova, and R.Z. Sagdeev, AIP Conf. Proc. 1320, 104 (2011).

${ }^{31}$ C.A.J. Palmer, J. Schreiber, S.R. Nagel, N.P. Dover, C. Bellei, F.N. Beg, S. Bott, R.J. Clarke, A.E. Dangor, S.M. Hassan, P. Hilz, D. Jung, S. Kneip, S.P.D. Mangles, K.L. Lancaster, A. Rehman, A.P.L. Robinson, C. Spindloe, J. Szerypo, M. Tatarakis, M. Yeung, M. Zepf, and Z. Najmudin, Phys. Rev. Lett. 108, 225002 (2012).

${ }^{32}$ T.-C. Liu, X. Shao, C.-S. Liu, M. He, B. Eliasson, V. Tripathi, J.-J. Su, J. Wang, and S.-H. Chen, New J. Phys. 15, 025026 (2013). 